\title{
Statistical Analysis of Residual Distribution in H.264 And A New Thought About Video Coding Algorithm ${ }^{1}$
}

\author{
Qian Qiao $^{2}$ and Wenhui Zhang ${ }^{3}$ and Na Sun ${ }^{4}$
}

\begin{abstract}
High computation complexity has significantly impacted H.264/AVC's application and promotion in real-time coding. As a result, how to reduce as much computation complexity as possible while ensuring high coding efficiency becomes an important subject in the study of video coding technology. Many scholars started their study from prediction of all-zero residual blocks to reduce the overhead of DCT, IDCT, quantization and inverse quantization, thus reduced the overall computation complexity. This paper analyzes the statistics of residual coefficient distribution based on the H.264 video coding standard, put forward a

\footnotetext{
1 This work was funded by the National Science and Technology Support Program: Stereoscopic Television Content Transmission Technology and Systems Research (2012BAH39F02)

2 Qian Qiao $(\bowtie)$

School of Information Engineering, Communication University of China, Beijing 100024

e-mail: qiaoqian628@gmail.com

Qian Qiao

School of Information Engineering, Communication University of China

Beijing, China

3 Wenhui Zhang

School of Information Engineering, Communication University of China

4 Na Sun

School of Information Engineering, Communication University of China

Beijing, China
} 
new idea about residual-based fast algorithms, and analyzes its feasibility by studying the experiment data.

Keywords: H.264 • Residual distribution • New idea • Fast Algorithm

\section{Introduction}

Video coding standards like MPEG2, MPEG4, H.263 and H.264 have seen their application go through the roof in multimedia field. Compared with the previous standards, the most outstanding advantage of H.264 is its high coding efficiency. It is estimated that to code a picture of the same quality level, H.264 can save over $50 \%$ of bitrates compared with other standards such as H.263. Yet the encoding computation complexity of H.264 is about 3 times as much as that of H.263, and the decoding computation complexity is about 2 times as much as H.263's. Hence in the study of video compression coding, making endeavour to reduce computation complexity is a key point that shouldn't be ignored. In the process of video coding, spatial redundancy is reduced through DCT, and temporal redundancy is reduced through predictive coding and motion compensation. Residual coding is the important content of video compression coding technology and responsible to process the data after prediction and write them into code stream and finally achieve further compression of video sequences. As the processing of residual data and related encoding make up the kernel of the code stream generating module, the property of relevant algorithms has tremendous influence on the performance of both encoder and decoder. This paper is carried out around the optimization algorithms of residual coding.

\section{Overview of 0-value Residual Prediction based Fast Algorithms}

At present many scholars have successively achieved computation reduction of DCT, quantization, inverse quantization and IDCT through prediction of 0 -value DCT coefficients, and that's a good way for H.264 algorithm optimization. In 
literature [1] the author used the relation between the SAD (Sum of Absolute Difference) of predicted residual block and quantization parameter (QP) to predict all-zero block. This method won't cause misjudgment, but it's efficiency is relatively low. The authors of literature [1] propose a new algorithm, take the SAD of each motion compensation block as the criteria to perform the all-zero block prediction. In literature [1], the author combines SAD with the DCT coefficients, deduces a more precise criteria to predict the all-zero blocks and improves the algorithm in literature [2]. Other literatures like [3] [4] [5] all propose algorithms of all-zero block prediction on the basis of study into DCT coefficient distribution models, thereby improving the encoding speed.

\section{New Ideas for Residual-based Fast Algorithms}

The theoretical foundation of 0 -value residual prediction is: when the quantization parameter is large, residuals after motion compensation in low rate $4 \times 4$ blocks tend to be very small after the DCT and quantization process, and there will be lots of all-zero coefficient blocks. Besides that there are a large number of all-zero blocks, if we can find out any other law in DCT residual distribution, new ideas for future research can be come up with.

Based on the theoretical foundation of residual distribution and all-zero block prediction, we suggest that there should be distribution law in the 16 or part of the 16 quantized DCT coefficients of a $4 \times 4$ block. That is to say, among all of the $4 \times 4$ quantized DCT coefficients, there may be several types appear more frequently. Then we may consider encoding these $4 \times 4$ block types that appear in most times and transmitting their serial numbers directly, while processing the other $4 \times 4$ QDCT (Quantized DCT) coefficients in conventional way. If this idea works, quite a lot of computation for processes such as entropy coding and run-length coding would be reduced. In section 5 of this paper, we'll conduct experiments based on this idea, and verify the feasibility of it by analyzing the experiment results. 


\section{Principle of DCT and Quantization}

In the advanced video compression standard H.264, a best mode is determined through comparison between several prediction modes, and the residuals of this best mode go through $4 \times 4$ integer discrete cosine transform to eliminate their spatial correlation. After that quantization parameters are determined according to the size of the image dynamic range, and then the DCT coefficients will be quantized according to the quantization parameter. Besides, if it is a chroma block or a intra $16 \times 16$ predicted luma block, its DC coefficients should be abstracted to form a $2 \times 2$ block (in the chroma block case) or a $4 \times 4$ block (in the intra $16 \times 16$ predicted luma block case) to go through the additional Hadamard transform, thus the rate could be further dropped [6]. Then run-length coding and entropy coding are performed on the quantized DCT coefficients, and the final compressed video stream is obtained.

\section{Statistical Analysis of the QDCT Coefficients in $4 \times 4$ Block}

The experiments use the reference code of H.264-- JM10.1 and four typical QCIF (Quarter Common Intermediate Format) video sequences-- Akiyo, Coastguard, Highway and Mobile, output the $4 \times 4$ block QDCT coefficients which are most frequently yielded, as well as their proportions of all blocks. GOP (Group of Picture) structure is IPPP, and QP is 28. One hundred frames of each sequence are coded.

The test sequences used in the experiments are as follows.

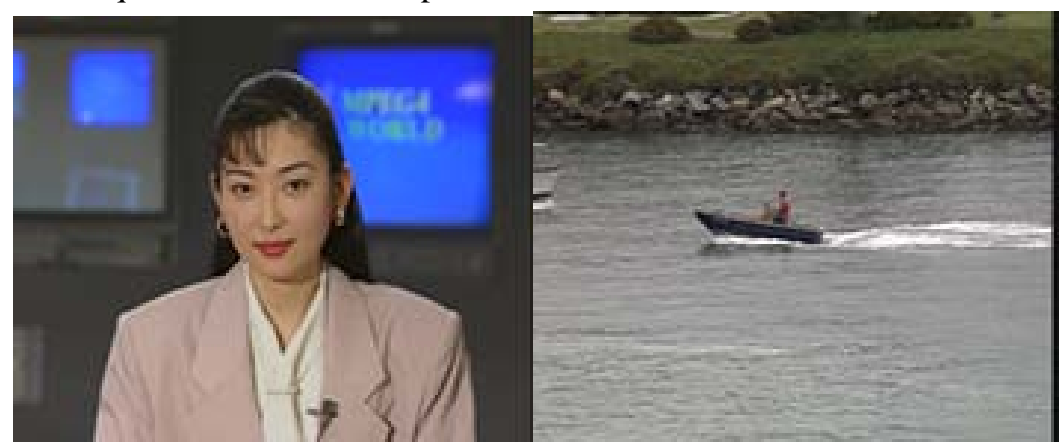

(a) Akiyo

(b) Coastguard 


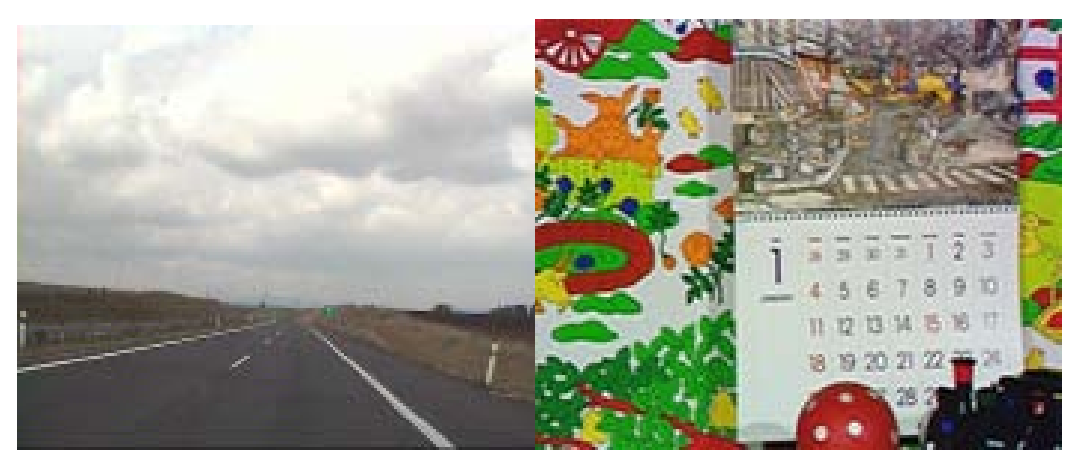

(c) Highway

(d) Mobile

Fig. 5.1 The test sequences used in the experiments

Table 5.1 The sequences used and their features

\begin{tabular}{|c|c|}
\hline Test sequence & Feature \\
\hline Akiyo & Still shot, Head movement \\
\hline Coastguard & Shift lens, Relative movement of objects \\
\hline Highway & Shift lens quickly from far to near \\
\hline Mobile & Shift lens, Various movements of several objects \\
\hline
\end{tabular}

Table 5.2 Result of sequence akiyo

\begin{tabular}{|c|c|c|c|c|c|c|c|c|c|c|c|c|c|c|c|c|c|}
\hline Type & \multicolumn{10}{|c|}{ Quantized residual } & Ratio \\
\hline 1 & 0 & 0 & 0 & 0 & 0 & 0 & 0 & 0 & 0 & 0 & 0 & 0 & 0 & 0 & 0 & 0 & $66.75 \%$ \\
\hline 2 & -1 & 0 & 0 & 0 & 0 & 0 & 0 & 0 & 0 & 0 & 0 & 0 & 0 & 0 & 0 & 0 & $4.09 \%$ \\
\hline 3 & 1 & 0 & 0 & 0 & 0 & 0 & 0 & 0 & 0 & 0 & 0 & 0 & 0 & 0 & 0 & 0 & $3.91 \%$ \\
\hline 4 & 0 & 1 & 0 & 0 & 0 & 0 & 0 & 0 & 0 & 0 & 0 & 0 & 0 & 0 & 0 & 0 & $1.33 \%$ \\
\hline
\end{tabular}

Table 5.2 (continued) Result of sequence akiyo

\begin{tabular}{|c|c|c|c|c|c|c|c|c|c|c|c|c|c|c|c|c|c|}
\hline Type & \multicolumn{10}{c|}{ Quantized residual } & \multicolumn{1}{c|}{ Ratio } \\
\hline 5 & 0 & -1 & 0 & 0 & 0 & 0 & 0 & 0 & 0 & 0 & 0 & 0 & 0 & 0 & 0 & 0 & $1.01 \%$ \\
\hline
\end{tabular}

Table 5.3 Result of sequence highway

\begin{tabular}{|c|c|c|c|c|c|c|c|c|c|c|c|c|c|c|c|c|c|}
\hline Type & \multicolumn{10}{|c|}{ Quantized residual } & Ratio \\
\hline 1 & 0 & 0 & 0 & 0 & 0 & 0 & 0 & 0 & 0 & 0 & 0 & 0 & 0 & 0 & 0 & 0 & $75.95 \%$ \\
\hline 2 & -1 & 0 & 0 & 0 & 0 & 0 & 0 & 0 & 0 & 0 & 0 & 0 & 0 & 0 & 0 & 0 & $6.96 \%$ \\
\hline 3 & 1 & 0 & 0 & 0 & 0 & 0 & 0 & 0 & 0 & 0 & 0 & 0 & 0 & 0 & 0 & 0 & $3.27 \%$ \\
\hline
\end{tabular}




\begin{tabular}{|c|c|c|c|c|c|c|c|c|c|c|c|c|c|c|c|c|c|}
\hline 4 & 0 & 0 & 1 & 0 & 0 & 0 & 0 & 0 & 0 & 0 & 0 & 0 & 0 & 0 & 0 & 0 & $1.37 \%$ \\
\hline 5 & 0 & 0 & -1 & 0 & 0 & 0 & 0 & 0 & 0 & 0 & 0 & 0 & 0 & 0 & 0 & 0 & $0.93 \%$ \\
\hline
\end{tabular}

Table 5.4 Result of sequence coastguard

\begin{tabular}{|c|c|c|c|c|c|c|c|c|c|c|c|c|c|c|c|c|c|}
\hline Type & \multicolumn{10}{|c|}{ Quantized residual } & Ratio \\
\hline 1 & 0 & 0 & 0 & 0 & 0 & 0 & 0 & 0 & 0 & 0 & 0 & 0 & 0 & 0 & 0 & 0 & $4.86 \%$ \\
\hline 2 & -1 & 0 & 0 & 0 & 0 & 0 & 0 & 0 & 0 & 0 & 0 & 0 & 0 & 0 & 0 & 0 & $3.69 \%$ \\
\hline 3 & 0 & 0 & 0 & 1 & 0 & 0 & 0 & 0 & 0 & 0 & 0 & 0 & 0 & 0 & 0 & 0 & $2.77 \%$ \\
\hline 4 & 0 & 0 & 1 & 0 & 0 & 0 & 0 & 0 & 0 & 0 & 0 & 0 & 0 & 0 & 0 & 0 & $2.56 \%$ \\
\hline 5 & 0 & 0 & -1 & 0 & 0 & 0 & 0 & 0 & 0 & 0 & 0 & 0 & 0 & 0 & 0 & 0 & $2.53 \%$ \\
\hline
\end{tabular}

Table 5.5 Result of sequence mobile

\begin{tabular}{|c|c|c|c|c|c|c|c|c|c|c|c|c|c|c|c|c|c|}
\hline Type & \multicolumn{10}{|c|}{ Quantized residual } & Ratio \\
\hline 1 & 0 & 0 & 0 & 0 & 0 & 0 & 0 & 0 & 0 & 0 & 0 & 0 & 0 & 0 & 0 & 0 & $45.52 \%$ \\
\hline 2 & -1 & 0 & 0 & 0 & 0 & 0 & 0 & 0 & 0 & 0 & 0 & 0 & 0 & 0 & 0 & 0 & $1.93 \%$ \\
\hline 3 & 0 & 0 & 0 & 0 & 0 & 0 & 0 & 0 & 0 & -1 & 0 & 0 & 0 & 0 & 0 & 0 & $1.32 \%$ \\
\hline 4 & 0 & 0 & 0 & 0 & 0 & 0 & 0 & 0 & 0 & 1 & 0 & 0 & 0 & 0 & 0 & 0 & $1.24 \%$ \\
\hline 5 & 0 & 0 & 0 & -1 & 0 & 0 & 0 & 0 & 0 & 0 & 0 & 0 & 0 & 0 & 0 & 0 & $1.07 \%$ \\
\hline
\end{tabular}

From the results of Table 2 Table 5, we can see that all-zero blocks take up the largest proportion of all quantized DCT coefficients, and that's why many literatures predict all-zero blocks to optimize algorithms for H.264. On the other hand, contrast of Table $2 \sim$ Table 5 also shows that for the sequences whose content is simple, the proportion of all-zero block is large (66.75\% for akiyo and $75.95 \%$ for highway); while for the sequences whose content is relatively complicated, the proportion is significantly decreased (48.6\% for coastguard and $45.52 \%$ for mobile). That is to say, all-zero block prediction can cause different results when used in different sequences' processing. Moreover, all-zero block prediction will introduce additional computation. Therefore, sequences' difference should be taken into account when deciding whether or not to use all-zero block prediction. In that case, it will be better if there is a more applicable algorithm. In addition to all-zero blocks, if there is any other QDCT block that tend to be yielded frequently, new idea for future research can be come up with-- 
transmitting the serial numbers of those types of blocks. The 5 most frequently yielded QDCT block types, namely type 1, 2, 3, 4, 5, were picked out. We can see from the charts that the not-all-zero blocks, namely types $2 \sim 5$, take up considerable proportion of all QDCT blocks. We can allocate a serial number for each of these $4 \times 4$ block types and transmit their numbers directly when they are to be encoded. Thus quite a number of computation for processes like entropy coding and run-length coding should be reduced. Yet the proportion of all these types is not so large, and the optimization result of this method may be consequently not so obvious. Then we consider looking for law in smaller blocks.

Table 5.6 4-Coefficient result of akiyo

\begin{tabular}{|c|c|c|c|c|c|c|c|c|c|c|c|}
\hline Type & \multicolumn{4}{|c|}{$\begin{array}{c}\text { Akiyo_4 coefficients } \\
\text { I }\end{array}$} & Ratio & Type & \multicolumn{4}{|c|}{$\begin{array}{c}\text { Akiyo_4 coefficients } \\
\text { II }\end{array}$} & Ratio \\
\hline 1 & 0 & 0 & 0 & 0 & $69.51 \%$ & 1 & 0 & 0 & 0 & 0 & $86.61 \%$ \\
\hline 2 & -1 & 0 & 0 & 0 & $4.56 \%$ & 2 & 0 & -1 & 0 & 0 & $1.29 \%$ \\
\hline 3 & 1 & 0 & 0 & 0 & $4.26 \%$ & 3 & 0 & 1 & 0 & 0 & $1.12 \%$ \\
\hline 4 & 0 & 1 & 0 & 0 & $1.76 \%$ & 4 & 1 & 0 & 0 & 0 & $1.07 \%$ \\
\hline 5 & 0 & -1 & 0 & 0 & $1.58 \%$ & 5 & -1 & 0 & 0 & 0 & $1.02 \%$ \\
\hline Type & \multicolumn{4}{|c|}{$\begin{array}{c}\text { Akiyo_4 coefficients } \\
\text { III } \\
\end{array}$} & Raito & Type & \multicolumn{4}{|c|}{$\begin{array}{c}\text { Akiyo_4 coefficients } \\
\text { IV }\end{array}$} & Ratio \\
\hline 1 & 0 & 0 & 0 & 0 & $94.63 \%$ & 1 & 0 & 0 & 0 & 0 & $96.83 \%$ \\
\hline 2 & -1 & 0 & 0 & 0 & $0.74 \%$ & 2 & -1 & 0 & 0 & 0 & $0.8 \%$ \\
\hline 3 & 1 & 0 & 0 & 0 & $0.62 \%$ & 3 & 1 & 0 & 0 & 0 & $0.73 \%$ \\
\hline 4 & 0 & 0 & 0 & 1 & $0.42 \%$ & 4 & 0 & 1 & 0 & 0 & $0.35 \%$ \\
\hline 5 & 0 & 0 & 0 & -1 & $0.36 \%$ & 5 & 0 & -1 & 0 & 0 & $0.26 \%$ \\
\hline
\end{tabular}

Table 5.7 4-Coefficient result of coastguard

\begin{tabular}{|c|c|c|c|c|c|c|c|c|c|c|c|}
\hline \multirow{2}{*}{ Type } & \multicolumn{5}{|c|}{ Coastguard_4 coefficients } & \multirow{2}{*}{ Ratio } & \multicolumn{3}{|c|}{ Type } & \multicolumn{5}{|c|}{ Coastguard_4 coefficients } & \multirow{2}{*}{ Ratio } \\
\hline 1 & 0 & 0 & 0 & 0 & $60.35 \%$ & 1 & 0 & 0 & 0 & 0 & $87.11 \%$ \\
\hline 2 & -1 & 0 & 0 & 0 & $5.04 \%$ & 2 & -1 & 0 & 0 & 0 & $3.07 \%$ \\
\hline 3 & 0 & 0 & 0 & 1 & $4.32 \%$ & 3 & 1 & 0 & 0 & 0 & $3.02 \%$ \\
\hline 4 & 0 & 0 & -1 & 0 & $3.87 \%$ & 4 & 0 & 0 & 0 & 1 & $0.94 \%$ \\
\hline 5 & 0 & 0 & 1 & 0 & $3.83 \%$ & 5 & 0 & 0 & 0 & -1 & $0.9 \%$ \\
\hline
\end{tabular}




\begin{tabular}{|c|c|c|c|c|c|c|c|c|c|c|c|}
\hline \multirow{2}{*}{ Type } & \multicolumn{3}{|c|}{ Coastguard_4 coefficients } & \multirow{2}{*}{ Ratio } & \multicolumn{3}{|c|}{ Type } & \multicolumn{5}{|c|}{ Coastguard_4 coefficients } & \multirow{2}{*}{ Ratio } \\
\hline 1 & 0 & 0 & 0 & 0 & $78.33 \%$ & 1 & 0 & 0 & 0 & 0 & $97.97 \%$ \\
\hline 2 & 0 & -1 & 0 & 0 & $4.45 \%$ & 2 & 0 & 0 & 1 & 0 & $0.46 \%$ \\
\hline 3 & 0 & 1 & 0 & 0 & $4.19 \%$ & 3 & 0 & 0 & -1 & 0 & $0.43 \%$ \\
\hline 4 & -1 & 0 & 0 & 0 & $2.28 \%$ & 4 & 1 & 0 & 0 & 0 & $0.27 \%$ \\
\hline 5 & 1 & 0 & 0 & 0 & $2.26 \%$ & 5 & -1 & 0 & 0 & 0 & $0.27 \%$ \\
\hline
\end{tabular}

Table 5.8 4-Coefficient result of highway

\begin{tabular}{|c|c|c|c|c|c|c|c|c|c|c|c|}
\hline Type & \multicolumn{4}{|c|}{$\begin{array}{c}\text { Highway_4 coefficients } \\
\text { I }\end{array}$} & Ratio & Type & \multicolumn{4}{|c|}{$\begin{array}{c}\text { Highway_4 coefficients } \\
\text { II }\end{array}$} & Ratio \\
\hline 1 & 0 & 0 & 0 & 0 & $78.63 \%$ & 1 & 0 & 0 & 0 & 0 & $96.5 \%$ \\
\hline 2 & -1 & 0 & 0 & 0 & $7.23 \%$ & 2 & -1 & 0 & 0 & 0 & $0.64 \%$ \\
\hline 3 & 1 & 0 & 0 & 0 & $3.41 \%$ & 3 & 1 & 0 & 0 & 0 & $0.63 \%$ \\
\hline 4 & 0 & 0 & 1 & 0 & $1.57 \%$ & 4 & 0 & -1 & 0 & 0 & $0.23 \%$ \\
\hline 5 & 0 & 0 & -1 & 0 & $1.11 \%$ & 5 & 0 & 0 & 0 & 1 & $0.2 \%$ \\
\hline Type & \multicolumn{4}{|c|}{$\begin{array}{c}\text { Highway_4 coefficients } \\
\text { III }\end{array}$} & Ratio & Type & \multicolumn{4}{|c|}{$\begin{array}{c}\text { Highway_4 coefficients } \\
\text { IV }\end{array}$} & Ratio \\
\hline 1 & 0 & 0 & 0 & 0 & $96.13 \%$ & 1 & 0 & 0 & 0 & 0 & $97.75 \%$ \\
\hline 2 & 0 & 1 & 0 & 0 & $0.52 \%$ & 2 & 0 & 0 & 1 & 0 & $0.23 \%$ \\
\hline 3 & 0 & -1 & 0 & 0 & $0.43 \%$ & 3 & 0 & 0 & -1 & 0 & $0.22 \%$ \\
\hline 4 & -1 & 0 & 0 & 0 & $0.3 \%$ & 4 & 1 & 0 & 0 & 0 & $0.19 \%$ \\
\hline 5 & 1 & 0 & 0 & 0 & $0.3 \%$ & 5 & 0 & 0 & 0 & 1 & $0.18 \%$ \\
\hline
\end{tabular}

Table 5.9 4-Coefficient result of mobile

\begin{tabular}{|c|c|c|c|c|c|c|c|c|c|c|c|}
\hline \multirow{2}{*}{ Type } & \multicolumn{5}{|c|}{ Mobile_4 coefficients } & Ratio & Type & \multicolumn{3}{c|}{ Mobile_4 coefficients } & Ratio \\
\hline 1 & 0 & 0 & 0 & 0 & $68.32 \%$ & 1 & 0 & 0 & 0 & 0 & $75.87 \%$ \\
\hline 2 & -1 & 0 & 0 & 0 & $3.69 \%$ & 2 & 1 & 0 & 0 & 0 & $2.56 \%$ \\
\hline 3 & 0 & 0 & 0 & 1 & $2.96 \%$ & 3 & -1 & 0 & 0 & 0 & $2.55 \%$ \\
\hline 4 & 0 & 0 & 0 & -1 & $2.75 \%$ & 4 & 0 & 0 & 1 & 0 & $1.9 \%$ \\
\hline 5 & 0 & 0 & -1 & 0 & $2.35 \%$ & 5 & 0 & 0 & -1 & 0 & $1.88 \%$ \\
\hline Type & \multicolumn{3}{|c|}{ Mobile_4 coefficients } & Ratio & Type & Mobile_4 coefficients & Ratio \\
\hline
\end{tabular}




\begin{tabular}{|c|c|c|c|c|c|c|c|c|c|c|c|}
\hline & \multicolumn{5}{|c|}{ III } & & \multicolumn{3}{|c|}{ IV } & \\
\hline 1 & 0 & 0 & 0 & 0 & $71.57 \%$ & 1 & 0 & 0 & 0 & 0 & $84.97 \%$ \\
\hline 2 & 0 & -1 & 0 & 0 & $3.58 \%$ & 2 & 1 & 0 & 0 & 0 & $2.06 \%$ \\
\hline 3 & 0 & 1 & 0 & 0 & $3.5 \%$ & 3 & -1 & 0 & 0 & 0 & $1.98 \%$ \\
\hline 4 & 1 & 0 & 0 & 0 & $2.27 \%$ & 4 & 0 & 0 & 1 & 0 & $1.87 \%$ \\
\hline 5 & -1 & 0 & 0 & 0 & $2.27 \%$ & 5 & 0 & 0 & -1 & 0 & $1.8 \%$ \\
\hline
\end{tabular}

In table6 table9, we classified the 16 QDCT coefficients of each $4 \times 4$ block into four groups respectively named I, II, III, IV, each of which contained four sequent coefficients. 5 of the 4-coefficient block types that occur most were picked out, and their proportions were listed. We can see from the charts that in whichever group, the all-zero groups, namely type 1 , take up the largest proportion of all 4-coefficient blocks. And compared with the all-zero $4 \times 4$ block types, all-zero 4-coefficient block takes up a much larger proportion. The not-all-zero ones, namely types $2 \sim 5$, take up considerable proportion of all 4-coefficient blocks.

It can be seen from the tables that in the selected four test sequences which include a total of 400 frames, after division the $4 \times 4$ block coefficient values are mainly concentrated on $0000,-1000, \cdots \cdots, 000-1$ the 9 types. In this case the classification numbering transmission mode is very suitable for the coefficients, and we can use 4 bits to code these nine types and transmit only their serial numbers. In this way, entropy coding for these 9 coefficient types can be eliminated. As a result, we can improve the compression efficiency at the same time of reducing computation complexity, thus better compression result is achieved.

\section{Conclusions}

This paper conducted detailed statistical analysis of quantized DCT coefficients on the basis of previous studies into residual distribution and all-zero block prediction, and proposed a new idea of fast algorithm. That is, to allocate serial numbers for the most frequently yielded $4 \times 4$ block types and transmit their numbers directly when they are to be encoded. Through the analysis of experiment 
results, we found that besides all-zero ones, there are other types of blocks which are frequently yielded. Moreover, the four-coefficient block types demonstrate better concentricity than the not divided $4 \times 4$ block types, so we can code these types of high frequency (For this experiment, 4-bit coding is sufficient) and transmit the serial number of each type. In this way, computation complexity of entropy coding and run-length coding can be reduced and higher compression efficiency can be achieved at the same time. This is the result obtained from theoretical analysis, we can also implement this idea with specific algorithms, and verify the correctness of this idea by the actual data comparison.

\section{$7 \quad$ References}

1. Zhou X, Yu. Z, Yu. S. Method for detecting all-zero DCT coefficients ahead of discrete cosine transformation and quantization[J]. Electron Lett,1998. 34(19): 1839-1840.

2. L. A. Sousa, "General method for eliminating redundant computations in video coding" Electron. Lett. vol. 36, pp. 306-307, 2000.

3. Pao I M, Sun M T. Modelling DCT coefficients for fast video encoding[J]. IEEE Transactions on Circuits and Systems for Video Technology, 1999, 9(4): 608-616.

4. Zhengguang Xie, Yong Liu, James Liu, Member, IEEE, and Tiejun Yang, A General Method for Detecting All-Zero Blocks Prior to DCT and Quantization, IEEE Transactions on Circuits and Systems for Video Technology, 2007,VOL. 17, NO. 2

5. Wang H. Kwong S. Hybrid model to detect zero quantized DCT coefficient in H.264[J]. IEEE Trans on Multimedia, 2007, 4(9): 728. 734.

6. Houjie Bi. A new generation of video compression coding standard. Peking: Posts and Telecom Press, 2005: 111-112 